\title{
RESPONSE OF THREE SUGAR BEET VARIETIES TO PLANTING DENSITY AND POTASSIUM FERTILIZER LEVELS UNDER SANDY SOIL CONDITIONS
}

\author{
Mohamed A.T. Yasin* \\ Agron. Dept., Fac. Agric., Zagazig University, Egypt
}

Received: 19/02/2017 ; Accepted: 23/03/2017

\begin{abstract}
Two field experiments were carried out at El-Khattara experimental farm, Faculty of Agriculture, Zagazig University, Sharkia Governorate, Egypt during the two successive seasons of $2011 / 2012$ and 2012/2013. This study aimed to investigate the response of yield and quality of three multigerm sugar beet varieties (Rass Poly, Panther and Pleno) to three planting densities (28000, 33600 and 42000 plants/fad.) and three potassium fertilizer levels ( 0,24 and $48 \mathrm{Kg} \mathrm{K}_{2} \mathrm{O} / \mathrm{fad}$.) in sandy soil under drip irrigation system. The combined analysis results showed that Pleno variety surpassed the other two investigated varieties in root length and diameter, fresh root weight/plant, root and recoverable sugar yields/fad. In addition, its roots contained lower percentages of $\mathrm{Na}$ and $\mathrm{K}$. Planting density affected on all traits, where increasing plant density up to 42000 plants/fad., significantly decreased root length and diameter, fresh top and root weights/plant, $\mathrm{Na}, \mathrm{K}$, alpha amino-N percentages and sugar loss to molasses (\%). On the other side, sucrose (\%), purity (\%), extractable sugar (\%), top, root and recoverable sugar yields were significantly and gradually increased. Moreover, root length, sucrose, $\mathrm{Na}, \mathrm{K}, \alpha$-amino-N, extractable sugar percentages, sugar loss to molasses (\%) (SLM) top, root and recoverable sugar yields were significantly increased by adding 24 $\mathrm{kg} \mathrm{K}_{2} \mathrm{O} /$ fad., while root diameter and fresh root weight/plant were significantly responded up to $48 \mathrm{~kg}$ $\mathrm{K}_{2} \mathrm{O} /$ fad. The interaction between the studied factors revealed that the maximum root and recoverable sugar yields/fad., could be obtained by planting Pleno variety with the dense planting of 42000 plants/ fad., and applying $24 \mathrm{~kg} \mathrm{~K}_{2} \mathrm{O} /$ fad.
\end{abstract}

Key words: Sugar beet varieties, planting density, K fertilization, yield and quality.

\section{INTRODUCTION}

Sugar beet (Beta vulgaris L.) is one of the most important sugar crops in Egypt. However it is well adapted to various Egyptian environmental conditions especially in newly reclaimed soils at North of Egypt due to its salinity tolerance. Sugar beet acreage in Egypt reached 508334 fad., which produced 11.05 million ton roots (FAOSTAT, 2016).

Increasing sugar beet productivity and quality could be achieved by selecting high yielding varieties. Many investigators scored significant differences among sugar beet varieties in most studied traits (Osman et al., 2003; El-Bakary,

\footnotetext{
${ }^{*}$ Corresponding author: Tel. : +201000843169

E-mail address: taha_agronomy_1978@yahoo.com
}

2006; Azzazy et al., 2007; El-Sheikh et al., 2009; Safina and Abdel Fatah, 2011; Shalaby et al., 2011; Aly et al., 2012; Al-Sayed and Attaya, 2015; Enan et al., 2016).

Planting density is very important factor that affect yield and quality of sugar beet. In this manner, Leilah et al. (2005), El-Geddawy et al. (2006), Nafei et al. (2010), El-Ghareib et al. (2012) and El-Hity et al. (2014) found that, the highest root, top and sugar yields per fad., root length and diameter, fresh weight/plant, sucrose $(\%)$ and purity $(\%)$ were obtained with the planting densities of 48000, 46666, 42000, 56000 and 52000 plants per fad., respectively. However, Sarhan et al. (2012) reported that, 
sowing sugar beet with planting density of 28000 plants per fad., recorded the highest averages of root length and diameter, root and top fresh weights/plant while using planting density of 46000 plants per fad., gave the highest averages of sucrose and purity (\%). On the other hand, the highest yields of root and sugar were achieved by using 35000 plants per fad. According to Varga et al. (2015) the plants grown at wider intra-row spacing (17 and 19 $\mathrm{cm}$ ) had a higher average of root weight than narrower intra-row spacing $(13$ and $15 \mathrm{~cm})$.

Potassium plays a vital role in regulating osmotic potential, increasing water uptake ability of sugar beet plants (Rengel and Damon, 2008; Zengin et al., 2009). Many researchers studied the effect of varying levels of $\mathrm{K}$ fertilizer on sugar beet grown in various soils. The maximum sugar loss and sucrose percentages were obtained by adding the highest $\mathrm{K}$ level i.e., $48 \mathrm{~kg} \mathrm{~K}_{2} \mathrm{O}$ per fad., (Abdel-Motagally and Attia, 2009). Increasing $K$ fertilizer level up to 36,42 and $59 \mathrm{~kg} \mathrm{~K} \mathrm{~K}_{2} \mathrm{O}$ per fad., significantly increased sugar beet root and top yields and impure sugar (\%) as well as pure sugar yield (Nafei et al., 2010, Mehrandish et al., 2012; El-Sarag and Moselhy, 2013), respectively. Raising K levels from 0 to $48 \mathrm{~kg} \mathrm{~K}_{2} \mathrm{O}$ per fad., significantly increased root fresh weight, root length and diameter, root yield (ton/ fad.) and sugar loss to molasses (\%). Otherwise, root content of sodium and $\alpha$-amino nitrogen were significantly decreased due to increasing $\mathrm{K}$ level up to $48 \mathrm{~kg} \mathrm{~K}_{2} \mathrm{O}$ per fad., (Abo-Shady et al., 2010). Also, Abdelaal et al. (2015) showed that, K fertilization at rate of 48 $\mathrm{kg} \mathrm{K}_{2} \mathrm{O}$ per fad., gave the highest averages of root length and diameter, $\alpha$-amino-N, $\mathrm{Na}$ and $\mathrm{K}$ as well as root and sugar yields per fad. In the contrary, a gradual reduction in sucrose (\%) had been detected with the increase in $\mathrm{K}$ level up to $36 \mathrm{~kg} \mathrm{~K}{ }_{2} \mathrm{O}$ per fad. Recently, Merwad (2016) revealed that top, root and recoverable sugar yield/ha, sucrose (\%) and purity (\%) were significantly increased, on the other hand, $\mathrm{K}$ $(\%), \quad \mathrm{Na}(\%)$ and $\alpha$-amino-N (\%) were significantly decreased due to raising $\mathrm{K}$ fertilizer level up to $200 \mathrm{~kg} \mathrm{~K} 2 \mathrm{O} / \mathrm{ha}$.

The objective of this work aimed to study the response of yield and quality of three multigerm sugar beet varieties to planting densities and potassium fertilizer levels under sandy soil conditions.

\section{MATERIALS AND METHODS}

In order to investigate the response of yield and its attributes as well as quality of some multigerm sugar beet (Beta vulgaris $\mathrm{L}$.) varieties grown in sandy soil to planting densities and potassium fertilizer levels under drip irrigation system, two field experiments were performed at Agricultural Research Station, Faculty of Agriculture, Zagazig University at El-Khattara region, Sharkia Governorate, Egypt during the two successive seasons 2011/2012 and 2012/ 2013. Soil samples were collected from the experimental sites at the depth of $0-30 \mathrm{~cm}$ before planting to determine soil mechanical and chemical properties. The mechanical and chemical analyses of the experimental field soil in the two seasons are presented in Table 1. A split-split plot design with three replicates was used. The main plots were occupied by sugar beet varieties (Rass Poly, Panther and Pleno). The sub-plots were devoted to planting densities (28000, 33600 and 42000 plants/fad.), while, potassium fertilizer levels $\left(0,24\right.$ and $48 \mathrm{~K}_{2} \mathrm{O} /$ fad.) were randomly distributed in the sub-sub plots. Each experiment included 27 treatments which were the combinations of three sugar beet varieties, three planting densities and three levels of potassium fertilizer. Each sub plot $\left(15 \mathrm{~m}^{2}\right)$ contained 6 drip irrigation lines, $5 \mathrm{~m}$ long $50 \mathrm{~cm}$ apart. Seeds of sugar beet were planted at distance of 30,25 and $20 \mathrm{~cm}$ between hills to obtain 28000,33600 and 42000 plants/ fad., respectively. Potassium fertilizer at the studied levels in the form of potassium sulphate $\left(48 \% \mathrm{~K}_{2} \mathrm{O}\right)$ was applied in two equal doses; the first was applied just after thinning, while the second was applied 15 days later.

Phosphorus fertilizer was added during seed bed preparation at level of $31 \mathrm{~kg} \mathrm{P}_{2} \mathrm{O}_{5} /$ fad., in the form of calcium superphosphate $(15.5 \%$ $\mathrm{P}_{2} \mathrm{O}_{5}$ ). Nitrogen fertilizer at rate of $120 \mathrm{~kg} \mathrm{~N} /$ fad., in the form of urea $(46.5 \% \mathrm{~N})$ was fertigated at five equal doses, the first was applied after thinning and the others were applied at 14 days intervals after the first application. In both seasons, the preceding crop was corn (Zea mays L.). Planting was done on 16 and 28 of October in the first and the second seasons, respectively. Manual planting was applied in hills with approximately 3-4 seeds per 
Table 1. Soil mechanical and chemical analyses of experimental sites and compost nutrients content in the two seasons

\begin{tabular}{|c|c|c|}
\hline Soil properties & 2012 & 2013 \\
\hline \multicolumn{3}{|l|}{ Mechanical analyses } \\
\hline Sand $(\%)$ & 86.52 & 91.5 \\
\hline Silt (\%) & 3.06 & 1.71 \\
\hline Clay $(\%)$ & 10.42 & 6.79 \\
\hline Organic matter $(\%)$ & 0.47 & 0.29 \\
\hline Soil texture & Loamy sand & Sandy \\
\hline \multicolumn{3}{|l|}{ Chemical analyses } \\
\hline $\mathrm{pH}$ & 7.96 & 8.09 \\
\hline $\mathrm{EC}$ mmhose/cm & 0.93 & 1.34 \\
\hline Available N (ppm) & 17.72 & 12.25 \\
\hline Available P (ppm) & 18.47 & 12.02 \\
\hline Available K (ppm) & 41.06 & 37.18 \\
\hline \multicolumn{3}{|c|}{ Soluble cations (meq/100 g) } \\
\hline $\mathrm{Na}^{+}$ & 0.68 & 1.04 \\
\hline $\mathrm{K}^{+}$ & 0.19 & 0.14 \\
\hline $\mathrm{Ca}^{++}$ & 43.21 & 22.80 \\
\hline $\mathrm{Mg}^{++}$ & 0.18 & 0.0 .13 \\
\hline \multicolumn{3}{|c|}{ Soluble anions (meq/100 g) } \\
\hline $\mathrm{Cl}^{-}$ & 0.64 & 0.79 \\
\hline $\mathrm{CO}_{3}^{--}$ & - & - \\
\hline $\mathrm{HCO}_{3}^{-}$ & 0.22 & 0.24 \\
\hline $\mathrm{SO}_{4}^{--}$ & 0.57 & 0.41 \\
\hline
\end{tabular}

Source: Central laboratory, Faculty of Agriculture, Zagazig University, Egypt.

hill and then plants were thinned at the fourth leaf stage (35 days from sowing). Drip irrigation system using underground water (around 900 ppm of total salts) and dripping time every 5 days was applied. Plants were kept free from weeds by hand hoeing for three times. The other regular agronomic practices, except the studied factors were done as recommended during growth seasons.

\section{Studied Characters}

\section{Root yield and its attributes}

At harvest (195 days after sowing) five plants were randomly taken from the second inner row of each plot, cleaned, thereafter roots were separated and weighed in kilograms to determine the following yield attributes: 
Root length $(\mathrm{cm})$, root diameter $(\mathrm{cm})$, top fresh weigh/plant (g) and root fresh weight/plant (g). All plants of the third, fourth and fifth central rows of each plot $\left(7.5 \mathrm{~m}^{2}\right)$ were harvested to estimate root yield (ton/fad.), top yield (ton/fad.) and recoverable sugar yield (ton/ fad.) $=$ Root yield $($ ton/fad. $) \times$ extractable sugar (\%) according to Mohamed (2002).

\section{Quality parameters}

Sucrose percentage (\%) was determined using polarimeter on a lead acetate extract of fresh macerate root as well as, impurities ( $\mathrm{Na}, \mathrm{K}$ and alpha amino nitrogen) were determined according to AOAC (2005). Purity percentage (\%) was calculated according to Devillers (1988) following this equation:

Purity $=99.36-(14.27(\mathrm{Na}+\mathrm{K}+\alpha$-amino nitrogen $) /$ sucrose \%). Sugar loss to molasses (SLM \%) $=$ $0.14(\mathrm{Na}+\mathrm{K})+0.25(\alpha$-amino nitrogen $)+0.50$, was determined according to Devillers (1988). Extractable sugar percentage (\%) was determined according to Dexter et al. (1967) following this equation: Extractable sugar percentage $(\%)=$ Sucrose $\%$ - SLM $\%-0.60)$.

\section{Statistical Analysis}

Data of the two successive seasons and their combined analysis were statistically analyzed according to Gomez and Gomez (1984). Treatment means were compared using least significant differences (LSD) test at 0.05 level of probability (Steel et al., 1997). Statistical analysis was performed by using analysis of variance technique of (MSTAT-C 1991) computer software package. The error mean squares of split split-plot design were homogenous (Bartlett's test), therefore, the combined analysis was calculated for all the studied characters in both seasons.

\section{RESULTS AND DISCUSSION}

\section{Varietal Differences}

\section{Root yield attributes}

Results presented in Table 2 exhibit significant differences among sugar beet varieties in root length, root diameter and fresh root weight/plant, while fresh top weight/plant could not reach to the significance level.
Meanwhile, Pleno variety produced the longest root $(25.83 \mathrm{~cm})$, as well as the longest root diameter $(14.23 \mathrm{~cm})$ and the heaviest fresh root weight/plant (2044.85 g) compared with the other two varieties (Rass Poly and Panther), according to combined results. In this connection, El-Bakary (2006), Azzazy et al. (2007) and ElSheikh et al. (2009) reported that sugar beet varieties significantly differed in root length and diameter and root fresh weight/plant. Also, the obtained results are in accordance with those reported by Safina and Abdel Fatah (2011), AlSayed and Attaya (2015) and Enan et al. (2016).

\section{Quality parameters}

The presented results in Tables 3 and 4 reveal that the investigated sugar beet varieties differed significantly in juice quality traits included $\mathrm{Na}(\%)$ and $\mathrm{K}(\%)$. Meanthrough the highest value of $\mathrm{Na}(\%)$ was recorded by Rass Poly variety compared with the other two varieties, while Rass Poly and Panther gained higher K (\%) compared with Pleno variety during both growing seasons and their combined. At contrary, the three sugar beet varieties did not vary significantly in sucrose $(\%)$, alpha amino $\mathrm{N}(\%)$, purity (\%), extractable sugar (\%) and SLM (\%), in spite of recording higher purity (\%) and extractable sugar (\%) and lower SLM (\%) by Pleno variety compared with the other two varieties. The obtained results are in agreement with those reported by Safina and Abdel Fatah (2011), Aly et al. (2012) and Enan et al. (2016). Also, Al-Sayed and Attaya (2015) reported that sugar beet varieties significantly differed in juice purity and sucrose percentages.

\section{Top yield (ton/fad.)}

Results in Table 5 show that the investigated sugar beet varieties differed highly significantly only in the $2^{\text {nd }}$ season wherein both Pleno and Panther varieties obtained higher top yield/fad., compared with Rass Poly variety. However such trend did not reach the level of significance in the $1^{\text {st }}$ season and the combined analysis. The obtained results are in concurrence with those stated by Osman et al. (2003), El-Bakary (2006), Azzazy et al. (2007), El-Sheikh et al. (2009) as well as Safina and Abdel Fatah (2011). In addition, Enan et al. (2016) recorded significant differences among sugar beet varieties in top yield/fad. 
Table 2. Root length $(\mathrm{cm})$, root diameter $(\mathrm{cm})$, fresh top weight/plant $(\mathrm{g})$ and fresh root weight/ plant (g) of sugar beet as affected by varietal differences, planting densities and potassium fertilizer levels during both growing seasons and their combined analysis

\begin{tabular}{|c|c|c|c|c|c|c|c|c|c|c|c|c|}
\hline \multirow{2}{*}{$\begin{array}{l}\text { Main effects and } \\
\text { interactions }\end{array}$} & \multicolumn{3}{|c|}{ Root length (cm) } & \multicolumn{3}{|c|}{ Root diameter $(\mathrm{cm})$} & \multicolumn{3}{|c|}{ Fresh top weight/plant (g) } & \multicolumn{3}{|c|}{ Fresh root weight/plant (g) } \\
\hline & $1^{\text {st }}$ & $2^{\text {nd }}$ & Comb. & $1^{\text {st }}$ & $2^{\text {nd }}$ & Comb. & $1^{\text {st }}$ & $2^{\text {nd }}$ & Comb. & $1^{\text {st }}$ & $2^{\text {nd }}$ & Comb. \\
\hline \multicolumn{13}{|l|}{ Varieties (V) } \\
\hline Rass Poly & $22.91 \mathrm{c}$ & $20.29 b$ & $21.60 \mathrm{c}$ & $12.42 \mathrm{~b}$ & $11.74 b$ & $12.08 \mathrm{c}$ & 517.22 & 526.52 & 521.87 & $1645.41 \mathrm{~b}$ & $1620.59 \mathrm{~b}$ & $1633.00 \mathrm{~b}$ \\
\hline Panther & $24.56 b$ & $21.46 \mathrm{~b}$ & $23.01 \mathrm{~b}$ & $13.84 \mathrm{ab}$ & $12.88 \mathrm{a}$ & $13.36 \mathrm{~b}$ & 527.30 & 557.19 & 542.24 & $1621.96 \mathrm{~b}$ & $1657.26 \mathrm{~b}$ & $1639.61 \mathrm{~b}$ \\
\hline Pleno & $26.96 \mathrm{a}$ & $24.70 \mathrm{a}$ & $25.83 \mathrm{a}$ & $15.10 \mathrm{a}$ & $13.35 \mathrm{a}$ & $14.23 \mathrm{a}$ & 594.60 & 581.89 & 588.24 & $2040.82 \mathrm{a}$ & $2048.89 a$ & $2044.85 \mathrm{a}$ \\
\hline F-test & $* *$ & $*$ & $* *$ & $*$ & $*$ & $* *$ & NS & NS & NS & $*$ & ** & $* *$ \\
\hline \multicolumn{13}{|l|}{ Planting densities (D) } \\
\hline 28000 plants/fad. & $25.89 \mathrm{a}$ & $23.73 \mathrm{a}$ & $24.81 \mathrm{a}$ & $14.53 \mathrm{a}$ & $13.54 \mathrm{a}$ & $14.03 \mathrm{a}$ & $650.44 \mathrm{a}$ & $645.44 \mathrm{a}$ & $647.94 \mathrm{a}$ & $1934.74 \mathrm{a}$ & $1869.70 \mathrm{a}$ & $1902.22 \mathrm{a}$ \\
\hline 33600 plants/fad. & $25.20 \mathrm{a}$ & $22.21 \mathrm{a}$ & $23.70 \mathrm{~b}$ & $14.12 \mathrm{a}$ & $12.97 \mathrm{a}$ & $13.54 \mathrm{~b}$ & $549.70 \mathrm{~b}$ & $564.59 \mathrm{~b}$ & $557.15 \mathrm{~b}$ & $1783.33 b$ & $1834.07 \mathrm{a}$ & $1808.70 \mathrm{~b}$ \\
\hline 42000 plants/fad. & $23.33 b$ & $20.52 b$ & $21.93 \mathrm{c}$ & $12.72 b$ & $11.46 \mathrm{~b}$ & $12.09 \mathrm{c}$ & $438.96 \mathrm{c}$ & $455.56 \mathrm{c}$ & $447.26 \mathrm{c}$ & $1590.11 \mathrm{c}$ & $1622.96 \mathrm{~b}$ & $1606.54 \mathrm{c}$ \\
\hline F-test & $* *$ & $* *$ & $* *$ & $* *$ & $* *$ & $* *$ & $* *$ & $* *$ & ** & $* *$ & $* *$ & $* *$ \\
\hline \multicolumn{13}{|c|}{ Potassium fertilizer level (K) } \\
\hline $0 \mathrm{~K}_{2} \mathrm{O}$ kg/fad. & $23.30 \mathrm{~b}$ & $19.73 \mathrm{c}$ & $21.52 \mathrm{~b}$ & $13.06 \mathrm{c}$ & $11.50 \mathrm{c}$ & $12.28 \mathrm{c}$ & 539.44 & 526.15 & 532.80 & $1634.22 \mathrm{~b}$ & $1605.19 \mathrm{c}$ & $1619.70 \mathrm{c}$ \\
\hline $24 \mathrm{~K}_{2} \mathrm{O} \mathrm{kg} / \mathrm{fad}$. & $25.48 \mathrm{a}$ & $22.75 b$ & $24.12 \mathrm{a}$ & $13.93 \mathrm{~b}$ & $12.70 \mathrm{~b}$ & $13.32 \mathrm{~b}$ & 550.11 & 558.67 & 554.39 & $1821.26 \mathrm{a}$ & $1786.59 \mathrm{~b}$ & $1803.93 \mathrm{~b}$ \\
\hline $48 \mathrm{~K}_{2} \mathrm{O} \mathrm{kg} / \mathrm{fad}$. & $25.64 \mathrm{a}$ & $23.97 \mathrm{a}$ & $24.81 \mathrm{a}$ & $14.37 \mathrm{a}$ & $13.77 \mathrm{a}$ & $14.07 \mathrm{a}$ & 549.56 & 580.78 & 565.17 & $1852.70 \mathrm{a}$ & $1934.96 \mathrm{a}$ & $1893.83 \mathrm{a}$ \\
\hline F-test & $* *$ & $* *$ & $* *$ & $* *$ & $* *$ & $* *$ & NS & NS & NS & ** & ** & $* *$ \\
\hline \multicolumn{13}{|l|}{ Interactions } \\
\hline $\mathbf{V} \times \mathbf{D}$ & $* *$ & NS & $* *$ & NS & NS & NS & NS & NS & NS & NS & NS & NS \\
\hline $\mathbf{V} \times \mathbf{K}$ & NS & $* *$ & $* *$ & $*$ & $* *$ & $*$ & NS & NS & NS & NS & NS & NS \\
\hline $\mathbf{D} \times \mathbf{K}$ & NS & NS & NS & NS & NS & NS & NS & NS & NS & NS & NS & NS \\
\hline
\end{tabular}

$*, * *$ and NS denote to significant at $0.05,0.01$ and not significant, respectively.

Table 3. Sucrose (\%), Na (\%), K (\%) and Alpha amino-N (\%) of sugar beet as affected by varietal differences, planting densities and potassium fertilizer levels during both growing seasons and their combined analysis

\begin{tabular}{|c|c|c|c|c|c|c|c|c|c|c|c|c|}
\hline \multirow[t]{2}{*}{ Main effects and interactions } & \multicolumn{3}{|c|}{ Sucrose (\%) } & \multicolumn{3}{|c|}{$\mathrm{Na}(\%)$} & \multicolumn{3}{|c|}{ K(\%) } & \multicolumn{3}{|c|}{ Alpha $\operatorname{amino} \mathbf{N}(\%)$} \\
\hline & $1^{\text {st }}$ & $2^{\text {nd }}$ & Comb. & $1^{\text {st }}$ & $2^{\text {nd }}$ & Comb. & $1^{\text {st }}$ & $2^{\text {nd }}$ & Comb. & $1^{\text {st }}$ & $2^{\text {nd }}$ & Comb. \\
\hline \multicolumn{13}{|l|}{ Varieties (V) } \\
\hline Rass Poly & 15.73 & 14.57 & 15.15 & $3.05 \mathrm{a}$ & $3.17 \mathrm{a}$ & $3.11 \mathrm{a}$ & $4.65 \mathrm{a}$ & $4.97 \mathrm{a}$ & $4.81 \mathrm{a}$ & 2.32 & 2.36 & 2.34 \\
\hline Panther & 15.39 & 14.25 & 14.82 & $2.69 \mathrm{~b}$ & $2.74 \mathrm{~b}$ & $2.71 \mathrm{~b}$ & $4.59 \mathrm{a}$ & $4.89 \mathrm{a}$ & $4.74 \mathrm{a}$ & 2.29 & 2.38 & 2.34 \\
\hline Pleno & 15.69 & 14.56 & 15.13 & $2.66 \mathrm{~b}$ & $2.83 \mathrm{~b}$ & $2.74 \mathrm{~b}$ & $4.07 \mathrm{~b}$ & $4.48 \mathrm{~b}$ & $4.28 \mathrm{~b}$ & 2.31 & 2.29 & 2.30 \\
\hline F-test & NS & NS & NS & $* *$ & $* *$ & $* *$ & $*$ & $*$ & $* *$ & NS & NS & NS \\
\hline \multicolumn{13}{|l|}{ Planting densities (D) } \\
\hline 28000 plants/fad. & $14.44 \mathrm{c}$ & $13.59 \mathrm{c}$ & $14.01 \mathrm{c}$ & $3.13 \mathrm{a}$ & $3.15 \mathrm{a}$ & $3.14 \mathrm{a}$ & $4.80 \mathrm{a}$ & $5.24 \mathrm{a}$ & $5.02 \mathrm{a}$ & $2.49 \mathrm{a}$ & $2.59 \mathrm{a}$ & $2.54 \mathrm{a}$ \\
\hline 33600 plants/fad. & $15.64 \mathrm{~b}$ & $14.41 \mathrm{~b}$ & $15.03 \mathrm{~b}$ & $2.90 \mathrm{~b}$ & $2.98 \mathrm{a}$ & $2.94 \mathrm{a}$ & $4.66 \mathrm{a}$ & $4.86 \mathrm{~b}$ & $4.76 \mathrm{a}$ & $2.33 \mathrm{~b}$ & $2.35 \mathrm{~b}$ & $2.34 \mathrm{~b}$ \\
\hline 42000 plants/fad. & $16.73 \mathrm{a}$ & $15.39 \mathrm{a}$ & $16.06 \mathrm{a}$ & $2.37 \mathrm{c}$ & $2.61 \mathrm{~b}$ & $2.49 \mathrm{~b}$ & $3.85 \mathrm{~b}$ & $4.24 \mathrm{c}$ & $4.05 \mathrm{~b}$ & $2.10 \mathrm{c}$ & $2.09 \mathrm{c}$ & $2.09 \mathrm{c}$ \\
\hline F-test & $* *$ & $* *$ & $* *$ & $* *$ & $* *$ & $* *$ & $* *$ & $* *$ & $* *$ & $* *$ & $* *$ & $* *$ \\
\hline \multicolumn{13}{|l|}{ Potassium fertilizer level (K) } \\
\hline $0 \quad \mathrm{~K}_{2} \mathrm{O} \mathrm{kg} / \mathrm{fad}$ & $14.45 \mathrm{~b}$ & $13.17 \mathrm{~b}$ & $13.81 \mathrm{~b}$ & $2.43 \mathrm{~b}$ & $2.69 \mathrm{~b}$ & $2.56 \mathrm{~b}$ & $3.85 \mathrm{c}$ & $4.34 \mathrm{~b}$ & $4.09 \mathrm{c}$ & $2.21 \mathrm{~b}$ & $2.11 \mathrm{~b}$ & $2.16 \mathrm{~b}$ \\
\hline $24 \mathrm{~K}_{2} \mathrm{O} \mathrm{kg} / \mathrm{fad}$. & $16.08 \mathrm{a}$ & $15.08 \mathrm{a}$ & $15.58 \mathrm{a}$ & $2.95 \mathrm{a}$ & $2.89 \mathrm{a}$ & $2.92 \mathrm{a}$ & $4.57 \mathrm{~b}$ & $4.92 \mathrm{a}$ & $4.74 \mathrm{~b}$ & $2.27 \mathrm{~b}$ & $2.42 \mathrm{a}$ & $2.34 \mathrm{a}$ \\
\hline $48 \mathrm{~K}_{2} \mathrm{O} \mathrm{kg} / \mathrm{fad}$. & $16.28 \mathrm{a}$ & $15.13 \mathrm{a}$ & $15.70 \mathrm{a}$ & $3.02 \mathrm{a}$ & $3.16 \mathrm{a}$ & $3.09 \mathrm{a}$ & $4.90 \mathrm{a}$ & $5.09 \mathrm{a}$ & $4.99 \mathrm{a}$ & $2.44 \mathrm{a}$ & $2.50 \mathrm{a}$ & $2.47 \mathrm{a}$ \\
\hline F-test & $* *$ & ** & $* *$ & $* *$ & $*$ & $* *$ & $* *$ & $* *$ & $* *$ & $* *$ & $* *$ & $* *$ \\
\hline \multicolumn{13}{|l|}{ Interactions } \\
\hline $\mathbf{V} \times \mathbf{D}$ & NS & NS & NS & NS & NS & NS & NS & NS & NS & NS & NS & NS \\
\hline $\mathbf{V} \times \mathbf{K}$ & NS & NS & NS & NS & NS & NS & NS & NS & NS & NS & NS & NS \\
\hline $\mathbf{D} \times \mathbf{K}$ & NS & NS & NS & NS & NS & NS & NS & NS & NS & NS & NS & NS \\
\hline
\end{tabular}

*,** and NS denote to significant at $0.05,0.01$ and not significant, respectively. 
Table 4. Purity (\%), extractable sugar (\%) and SLM (\%) of sugar beet as affected by varietal differences, planting densities and potassium fertilizer levels during both growing seasons and their combined analysis

\begin{tabular}{|c|c|c|c|c|c|c|c|c|c|}
\hline \multirow{2}{*}{$\begin{array}{l}\text { Main effects and } \\
\text { interactions }\end{array}$} & \multicolumn{3}{|c|}{ Purity (\%) } & \multicolumn{3}{|c|}{ Extractable sugar (\%) } & \multicolumn{3}{|c|}{ SLM (\%) } \\
\hline & $1^{\text {st }}$ & $2^{\text {nd }}$ & Comb. & $\mathbf{1}^{\mathrm{st}}$ & $2^{\text {nd }}$ & Comb. & $1^{\mathrm{st}}$ & $2^{\text {nd }}$ & Comb. \\
\hline \multicolumn{10}{|l|}{ Varieties (V) } \\
\hline Rass Poly & 90.20 & 88.99 & 89.60 & 12.97 & 11.74 & 12.36 & 2.16 & 2.23 & 2.19 \\
\hline Panther & 90.41 & 89.25 & 89.83 & 12.70 & 11.49 & 12.09 & 2.09 & 2.16 & 2.13 \\
\hline Pleno & 91.07 & 89.88 & 90.48 & 13.07 & 11.87 & 12.47 & 2.02 & 2.10 & 2.06 \\
\hline F-test & NS & NS & NS & NS & NS & NS & NS & NS & NS \\
\hline \multicolumn{10}{|l|}{ Planting densities (D) } \\
\hline 28000 plants/fad. & 89.07 & 87.77 & $88.42 \mathrm{c}$ & $11.61 \mathrm{c}$ & $10.67 \mathrm{c}$ & $11.14 \mathrm{c}$ & $2.23 \mathrm{a}$ & $2.32 \mathrm{a}$ & $2.28 \mathrm{a}$ \\
\hline 33600 plants/fad. & 90.34 & 89.26 & $89.80 \mathrm{~b}$ & $12.90 \mathrm{~b}$ & $11.62 \mathrm{~b}$ & $12.26 \mathrm{~b}$ & $2.14 \mathrm{a}$ & $2.18 \mathrm{a}$ & $2.16 \mathrm{a}$ \\
\hline 42000 plants/fad. & 92.28 & 91.08 & $91.68 \mathrm{a}$ & $14.23 \mathrm{a}$ & $12.81 \mathrm{a}$ & $13.52 \mathrm{a}$ & $1.90 \mathrm{~b}$ & $1.98 \mathrm{~b}$ & $1.94 \mathrm{~b}$ \\
\hline F-test & NS & NS & $*$ & $*$ & $*$ & $*$ & $*$ & * & $* *$ \\
\hline \multicolumn{10}{|c|}{ Potassium fertilizer level (K) } \\
\hline $0 \quad K_{2} O \mathrm{~kg} / \mathrm{fad}$ & 90.86 & 89.34 & 90.10 & $11.92 \mathrm{~b}$ & $10.56 \mathrm{~b}$ & $11.24 \mathrm{~b}$ & $1.93 \mathrm{~b}$ & $2.01 \mathrm{~b}$ & $1.97 \mathrm{~b}$ \\
\hline $24 \mathrm{~K}_{2} \mathrm{O} \mathrm{kg} / \mathrm{fad}$. & 90.61 & 89.62 & 90.12 & $13.36 \mathrm{a}$ & $12.29 \mathrm{a}$ & $12.82 \mathrm{a}$ & $2.12 \mathrm{a}$ & $2.20 \mathrm{a}$ & $2.16 \mathrm{a}$ \\
\hline $48 \mathrm{~K}_{2} \mathrm{O} \mathrm{kg} / \mathrm{fad}$. & 90.21 & 89.15 & 89.68 & $13.46 \mathrm{a}$ & $12.25 \mathrm{a}$ & $12.85 \mathrm{a}$ & $2.22 \mathrm{a}$ & $2.28 \mathrm{a}$ & $2.25 \mathrm{a}$ \\
\hline F-test & NS & NS & NS & $* *$ & $* *$ & $* *$ & $*$ & $*$ & $* *$ \\
\hline \multicolumn{10}{|l|}{ Interactions } \\
\hline $\mathbf{V} \times \mathbf{D}$ & NS & NS & NS & NS & NS & NS & NS & NS & NS \\
\hline $\mathbf{V} \times \mathbf{K}$ & NS & NS & NS & NS & NS & NS & NS & NS & NS \\
\hline $\mathbf{D} \times \mathbf{K}$ & NS & NS & NS & NS & $*$ & $* *$ & NS & NS & NS \\
\hline
\end{tabular}

$*, * *$ and NS denote to significant at $0.05,0.01$ and not significant, respectively.

Table 5. Top, root yields (ton/fad.) and recoverable sugar yield (ton/fad.) of sugar beet as affected by varietal differences, planting densities and potassium fertilizer levels during both growing seasons and their combined analysis

\begin{tabular}{|c|c|c|c|c|c|c|c|c|c|}
\hline \multirow[t]{2}{*}{$\begin{array}{l}\text { Main effects and } \\
\text { interactions }\end{array}$} & \multicolumn{3}{|c|}{ Top yield (ton/fad.) } & \multicolumn{3}{|c|}{ Root yield (ton/fad.) } & \multicolumn{3}{|c|}{$\begin{array}{c}\text { Recoverable sugar yield } \\
\text { (ton/fad.) }\end{array}$} \\
\hline & $1^{\text {st }}$ & $2^{\text {nd }}$ & Comb. & $1^{\text {st }}$ & $2^{\text {nd }}$ & Comb. & $1^{\text {st }}$ & $2^{\text {nd }}$ & Comb. \\
\hline \multicolumn{10}{|l|}{ Varieties (V) } \\
\hline Rass Poly & 14.40 & $11.93 \mathrm{~b}$ & 12.92 & $46.30 \mathrm{~b}$ & $42.77 \mathrm{~b}$ & $44.53 \mathrm{~b}$ & $6.05 \mathrm{~b}$ & $5.06 \mathrm{~b}$ & $5.56 \mathrm{~b}$ \\
\hline Panther & 16.33 & $13.20 \mathrm{a}$ & 14.76 & $46.86 \mathrm{~b}$ & $43.89 \mathrm{~b}$ & $45.38 \mathrm{~b}$ & $6.00 \mathrm{~b}$ & $5.07 \mathrm{~b}$ & $5.54 \mathrm{~b}$ \\
\hline Pleno & 16.62 & $14.03 \mathrm{a}$ & 15.33 & $55.04 \mathrm{a}$ & $53.58 \mathrm{a}$ & $54.31 \mathrm{a}$ & $7.29 \mathrm{a}$ & $6.41 \mathrm{a}$ & $6.85 \mathrm{a}$ \\
\hline F-test & NS & $* *$ & NS & $* *$ & $* *$ & $* *$ & $* *$ & $* *$ & $* *$ \\
\hline \multicolumn{10}{|l|}{ Planting densities (D) } \\
\hline 28000 plants/fad. & $14.29 \mathrm{~b}$ & $10.82 \mathrm{c}$ & $12.55 \mathrm{c}$ & $44.24 \mathrm{c}$ & $43.39 \mathrm{~b}$ & $43.81 \mathrm{c}$ & $5.16 \mathrm{c}$ & $4.65 \mathrm{c}$ & $4.91 \mathrm{c}$ \\
\hline 33600 plants/fad. & $16.06 \mathrm{a}$ & $13.07 \mathrm{~b}$ & $14.57 \mathrm{~b}$ & $49.04 \mathrm{~b}$ & $47.57 \mathrm{a}$ & $48.30 \mathrm{~b}$ & $6.35 \mathrm{~b}$ & $5.55 \mathrm{~b}$ & $5.95 \mathrm{~b}$ \\
\hline 42000 plants/fad. & $17.00 \mathrm{a}$ & $14.76 \mathrm{a}$ & $15.88 \mathrm{a}$ & $54.91 \mathrm{a}$ & $49.29 \mathrm{a}$ & $52.10 \mathrm{a}$ & $7.84 \mathrm{a}$ & $6.34 \mathrm{a}$ & $7.09 \mathrm{a}$ \\
\hline F-test & $* *$ & $* *$ & $* *$ & $* *$ & $* *$ & $* *$ & $* *$ & $* *$ & $* *$ \\
\hline \multicolumn{10}{|c|}{ Potassium fertilizer level (K) } \\
\hline $0 \mathrm{~K}_{2} \mathrm{O} \mathrm{kg} / \mathrm{fad}$ & 15.36 & $12.52 \mathrm{~b}$ & $13.94 \mathrm{~b}$ & $45.15 \mathrm{~b}$ & $43.34 \mathrm{~b}$ & $44.25 \mathrm{~b}$ & $5.43 \mathrm{~b}$ & $4.60 \mathrm{~b}$ & $5.01 \mathrm{~b}$ \\
\hline $24 \mathrm{~K}_{2} \mathrm{O} \mathrm{kg} / \mathrm{fad}$. & 15.98 & $13.10 \mathrm{a}$ & $14.54 \mathrm{a}$ & $50.60 \mathrm{a}$ & $48.09 \mathrm{a}$ & $49.34 \mathrm{a}$ & $6.80 \mathrm{a}$ & $5.94 \mathrm{a}$ & $6.37 \mathrm{a}$ \\
\hline $48 \mathrm{~K}_{2} \mathrm{O} \mathrm{kg} / \mathrm{fad}$. & 16.01 & $13.03 \mathrm{a}$ & $14.52 \mathrm{a}$ & $52.44 \mathrm{a}$ & $48.82 \mathrm{a}$ & $50.63 \mathrm{a}$ & $7.11 \mathrm{a}$ & $6.01 \mathrm{a}$ & $6.56 \mathrm{a}$ \\
\hline F-test & NS & $*$ & $*$ & $* *$ & $* *$ & $* *$ & $* *$ & $* *$ & $* *$ \\
\hline \multicolumn{10}{|l|}{ Interactions } \\
\hline $\mathbf{V} \times \mathbf{D}$ & NS & $*$ & NS & $* *$ & NS & $* *$ & $*$ & NS & $* *$ \\
\hline $\mathbf{V} \times \mathbf{K}$ & NS & $* *$ & NS & NS & NS & NS & NS & $*$ & NS \\
\hline $\mathbf{D} \times \mathrm{K}$ & NS & $*$ & NS & NS & $*$ & $*$ & $* *$ & $*$ & $* *$ \\
\hline
\end{tabular}




\section{Root yield (t/fad.)}

The results in Table 5 cleare that sugar beet varieties varied significantly in root yield, when Pleno variety produced the highest root yield/ fad., during both growing seasons and their combined analysis compared with the other two investigated varieties. These results followed the same patterns of root length, root diameter and fresh root weight/ plant (Table 2) which confirmed the superiority of Pleno variety in root yield attributes as well as in root yield/fad. The superiority of Pleno variety in root yield/fad., over Rass Poly and Panther varieties amounted to $21.96 \%$ and $19.68 \%$, respectively. These results are in line with those reported by Osman et al. (2003), El-Bakary (2006), Azzazy et al. (2007), El-Sheikh et al. (2009), Shalaby et al. (2011) and Aly et al. (2012). Also, Al-Sayed and Attaya (2015) recorded significant differences among sugar beet varieties in root yield during both seasons.

\section{Recoverable sugar yield (ton/fad.)}

Recoverable sugar yield/fad., as a main economical yield, was influenced by investigated sugar beet varieties (Table 5). The results followed the same patterns of above-mentioned root yield attributes as well as root yield/fad. Since, sugar beet varieties differed highly significantly during both growing seasons and their combined, where Pleno variety appeared to produce the highest recoverable sugar yield/fad., compared with the other two varieties. Respecting the combined results, the three sugar beet varieties Rass Poly, Panther and Pleno produced recoverable sugar yield valued 5.56, 5.54 and 6.85 ton/fad., respectively. Thus, Pleno variety gave relative increase reached to $23.20 \%$ and $23.65 \%$ over Rass Poly and Panther varieties, in the same respective order. Such varietal differences are mostly due to genetic potential variations as well as their interaction with the environmental conditions. The obtained results are in harmony with those stated by Osman et al. (2003), El-Bakary (2006), Azzazy et al. (2007), El-Sheikh et al. (2009), Shalaby et al. (2011), Aly et al. (2012) as well as Al-Sayed and Attaya (2015). On the other hand, Enan et al. (2016) showed no significant differences among sugar beet varieties in corrected sugar yield/fad.

\section{Impact of Planting Densities}

\section{Root yield attributes}

Results in Table 2 show that root performance in terms of root length, root diameter, fresh top and root weights/plant were highly significantly affected by planting density rates throughout both growing seasons and their combined. Decreasing planting density from 42000 to 33600 and 28000 plants/fad., tended to cause gradual increase in root yield attributes. Thus, the highest mean of each root length $(24.81 \mathrm{~cm})$, root diameter $(14.03 \mathrm{~cm})$, fresh top weight/plant $(647.94 \mathrm{~g})$ and fresh root weight/plant (1902.22 g) were achieved by the low planting density of 28000 plants/fad., according to the combined results. In other words, dense planting tended to decrease root dimensions i.e. length and diameter as well as fresh top and root weights/ plant. These results may explain that low planting density of 28000 plants/fad., minimize the inter competition between plants which led to high light use efficiency of solar radiation utilized by plants. In turn high in the conversion of light energy to chemical energy and consequently high accumulation of dry mater and increase of yield and its attributes. In this connection, Nafei et al. (2010) found that increasing plant spacing from 20 to $30 \mathrm{~cm}$ caused significant increase in root length, diameter and fresh weight/plant. The obtained results are in accordance with those reported by Leilah et al. (2005), El-Geddawy et al. (2006), Shalaby et al. (2011), El-Ghareib et al. (2012), Sarhan et al. (2012) and El-Hity et al. (2014).

\section{Quality parameters}

Results in Tables 3 and 4 exhibit significant differences effects due to planting densities on various juice quality parameters (sucrose (\%), $\mathrm{Na}(\%), \mathrm{K}(\%)$, alpha amino-N (\%), purity (\%), extractable sugar (\%) and SLM (\%) during both seasons and their combined analysis. Meanthrough, dense planting of 42000 plants/ fad., achieved the highest sucrose (\%), purity (\%) and extractable sugar (\%) as compared with intermediate or low planting densities. Otherwise, intermediate and low planting densities recorded higher $\mathrm{Na}(\%), \mathrm{K}(\%)$ and 
SLM (\%) compared with dense planting. However, low planting density of 28000 plants/ fad., gave the highest alpha amino-N (\%) compared with intermediate and dense planting densities. In this manner, Leilah et al. (2005) reported that sowing sugar beet on $25 \mathrm{~cm}$ between plants gave the highest sucrose (\%). Also, Nafei et al. (2010) showed that the highest sucrose (\%) and purity (\%) were obtained by dense planting of 42000 plants/fad. In the same trend, Shalaby et al. (2011) reported that increasing distance within plants increased $\mathrm{Na}$ $(\%)$ and $\mathrm{K}(\%)$. Also, the obtained results are in agreement with those reported by El-Ghareib et al. (2012) and El-Hity et al. (2014).

\section{Top yield (ton/fad.)}

As shown in Table 5, planting densities presented highly significant differences during both seasons and their combined, where the highest top yield/fad., was achieved by dense planting of 42000 plants/fad. Otherwise, the low planting density gave the lowest top yield/ fad. The obtained results are in harmony with those reported by Sarhan et al. (2012), El-Hity et al. (2014) as well as Neana and Abd El Hak (2014) who stated that top yield/unit area appeared to be gradually increased as plant density was increased.

\section{Root yield (ton/fad.)}

Results in Table 5 indicate that root yield/ fad., almost followed the same patterns of top yield/ fad, where dense planting of 42,000 plants/ fad., achieved the highest root yield/fad. Also, low density of 28,000 plants/fad recorded the lowest root yield/fad. It is worth to mention that these results followed the reverse directions with those obtained from aforementioned results of root and top traits/plant (Table 2). Where the low density of 28,000 plants/fad., with wider spacing of $30 \mathrm{~cm}$ between hills gave the highest values of those traits. Such results might be attributed to reducing inter-competition between plants rather than reducing intra-competition in dense planting with $20 \mathrm{~cm}$ between hills. The obtained results are in agreement with those reported by Shalaby et al. (2011), Aly et al. (2012), Sarhan et al. (2012), El-Hity et al. (2014) as well as Neana and Abd El Hak (2014).

\section{Recoverable sugar yield (ton/fad.)}

Recoverable sugar yield/fad., results as affected by planting density are presented in Table 5, results reveal highly significant differences during both growing seasons and their combined. Likely, the results followed the patterns of top and root yields/fad., in the same Table, as well as extractable sugar (\%) (Table 4) whereas dense planting of 42000 plants/fad., achieved the highest recoverable sugar yield/ fad., during both seasons and their combined. Generally, recoverable sugar yield tended to be gradually increased as planting density was increased from 28,000 and 33,600 to 42,000 plants/fad., with relative increase amounted to $44.40 \%$ and $19.16 \%$ in the same respective order, concerning the combined results. The superiority of dense planting above low and intermediate densities was expected since it had the highest means of root yield and extractable sugar (\%). Otherwise, the low density of 28000 plants/fad., recorded the lowest recoverable sugar yield/fad., as compared with dense and intermediate planting densities. Again, the superiority of dense planting with $20 \mathrm{~cm}$ between hills and $50 \mathrm{~cm}$ between drip irrigation lines may be attributed to less intra-competition between plants for light and nutrients. In this connection, Masri (2008) reported that increasing plant density from 35000 to 40000 plants/fad., significantly increased sugar yield by $21.3 \%$. In addition, El-Sarag (2009) found that the highest plant density (46000 plants/fad.) gave maximum sugar yield. Furthermore, Aly et al. (2012) showed that sugar yield/fad., was significantly increased with reducing spacing between hills. The obtained results are in harmony with those of Nafei et al. (2010), Shalaby et al. (2011), Sarhan et al. (2012) as well as Neana and Abd El Hak (2014).

\section{Impact of Potassium Fertilizer Levels}

\section{Root yield attributes}

Results in Table 2 clear that root length, root diameter and fresh root weight/plant were significantly affected by potassium fertilizer levels during both growing seasons and their combined, while the differences in fresh top weight/plant did not reach to the level of significance. Meanthrough, the application of either 24 or $48 \mathrm{~kg} \mathrm{~K}_{2} \mathrm{O} /$ fad., recorded higher 
means of root length than the control without significant differences between those two levels. However, the results of the second season confirmed by combined analysis showed that the high level of $\mathrm{K}\left(48 \mathrm{~kg} \mathrm{~K}_{2} \mathrm{O} /\right.$ fad.) recorded the longest root diameter and the heaviest fresh root weight/plant compared with control and $24 \mathrm{~kg}$ $\mathrm{K}_{2} \mathrm{O} /$ fad., applications. These results clearly show the positive effect of $\mathrm{K}$ fertilizer on sugar beet growth and development. Potassium plays an important role in regulating osmotic potential, increasing water uptake ability of sugar beet plants (Zengin et al., 2009). Furthermore, Nafei et al. (2010) reported that potassium fertilizer level at rate of $36 \mathrm{~kg} \mathrm{~K}_{2} \mathrm{O}$ / fad., gave significant increase in root length, root diameter and fresh root weight/ plant. AboShady et al. (2010) reported significant increase in root length, root diameter and fresh weight/plant due to raising potassium fertilizer level up to $48 \mathrm{~kg} \mathrm{~K}_{2} \mathrm{O} /$ fad. Also, Abdelaal et al. (2015) showed that, $\mathrm{K}$ fertilization at rate of 48 $\mathrm{kg} \mathrm{K}_{2} \mathrm{O}$ per fad., gave the highest averages of root length and diameter.

\section{Quality parameters}

The effect of potassium fertilizer levels on various juice quality traits is given in Tables 3 and 4 . The presented results recorded significant differences during both seasons and their combined, while it did not reach the level of significance for purity (\%). Meanthrough, according to combined results, the application of either 24 or $48 \mathrm{~kg} \mathrm{~K} \mathrm{~K}_{2} \mathrm{O} /$ fad., significantly increased sucrose (\%), Na (\%), K (\%), alpha amino-N (\%), extractable sugar (\%) and SLM (\%) with no significant differences between the two levels. The increment in impurities $(\mathrm{Na} \%$, $\mathrm{K} \%$ and alpha amino-N\%) and (SLM\%) due to raising potassium fertilizer level may be ascribed to the fact that high amounts of potassium in sugar beet roots decrease crystallization of sucrose in juice during sugar extraction and loss of sucrose in molasses. The obtained results are in accordance with those reported by Nafei et al. (2010), Mehrandish et al. (2012) and El-Sarag and Moselhy (2013). Further, Abdelaal et al. (2015) showed that, K fertilization at rate of $48 \mathrm{~kg} \mathrm{~K}_{2} \mathrm{O}$ per fad., gave the highest averages of $\alpha$-amino $\mathrm{N}(\%), \mathrm{Na}(\%)$ and $\mathrm{K}(\%)$ while, a gradual reduction in sucrose (\%) had been detected with the increase in $\mathrm{K}$ level over $36 \mathrm{~kg} \mathrm{~K} \mathrm{~K}_{2} \mathrm{O} /$ fad. However, Merwad (2016) reported that sucrose (\%) and purity (\%) were significantly increased but, $\mathrm{K}(\%), \mathrm{Na}(\%)$ and $\alpha$-amino-N (\%) were significantly decreased due to raising $\mathrm{K}$ fertilizer level up to $200 \mathrm{~kg}$ $\mathrm{K}_{2} \mathrm{O} / \mathrm{ha}$.

\section{Top yield (ton/fad.)}

Results in Table 5 show that potassium fertilizer caused significant differences in top yield/fad., during the second season and the combined, while the differences in the first season followed the same trend, but it could not reach the level of significance. The two application levels of potassium did not differed significantly regarding top yield/fad. At contrary, control treatment (without K application) recorded the lowest top yield/fad. These results show the positive effect of $\mathrm{K}$ on sugar beet growth and development, which in turn increased leaf area and chlorophyll content (Mubarak et al., 2016). The results almost followed the patterns of root yield attributes (Table 2). These results are in a good line with those reported by Nafie et al. (2010), Mehrandish et al. (2012), El-Sarag and Moselhy (2013) and Merwad (2016).

\section{Root yield (ton/fad.)}

Results in Table 5 indicate that application of potassium fertilizer significantly increased root yield/fad., during both growing seasons and their combined. This increase was confirmed by the two seasons and their combined with no significant differences between the two levels of potassium. These results followed the same patterns of top yield/fad., (Table 5) and the most of root yield attributes (Table 2). Potassium is essential for growth and it is the main element used to regulate the water content of the plant (Rengel and Damon, 2008). Nafei et al. (2010) reported that root yield was significantly increased by adding $36 \mathrm{~kg} \mathrm{~K} 2 \mathrm{O} /$ fad., compared with control treatment. This observation coincide with those found by Mehrandish et al. (2012), Abdelaal et al. (2015) and Merwad (2016).

\section{Recoverable sugar yield (ton/fad.)}

Results of recoverable sugar yield as influenced by potassium fertilizer levels are given in Table 5 which detected highly 
significant differences during both seasons and their combined. Meanwhile, the recorded results followed the patterns of top and root yields. Where the application of either 24 or $48 \mathrm{~kg} \mathrm{~K}_{2} \mathrm{O}$ / fad., increased recoverable sugar yield/fad., as compared with control treatment, with no significant differences between the two potassium fertilizer applied levels. Potassium levels of 0,24 and $48 \mathrm{~kg} \mathrm{~K}_{2} \mathrm{O} /$ fad., produced $5.01,6.37$ and 6.56 tones recoverable sugar yield/ fad., in the same respective order (according to the combined results). Thus, the superiority of application 24 and $48 \mathrm{~kg} \mathrm{~K}_{2} \mathrm{O}$ / fad., over control treatment reached relative increase amounted to 27.15 and $30.94 \%$ for the same respective order. Generally, the application of potassium fertilizer appeared to increase recoverable sugar yield significantly around $1.50 \mathrm{ton} / \mathrm{fad}$. This increase in root and sugar yields may be due to that potassium is a mobile element in the plant tissue and it plays an important role in photosynthesis through carbohydrate metabolism, osmotic regulation, nitrogen uptake, protein synthesis and translocation of assimilates (Ulgen et al., 2009; Nafei et al., 2010). The obtained results are in concurrence with those stated by Mehrandish et al. (2012), El-Sarag and Moselhy (2013), Abdelaal et al. (2015) and Merwad (2016).

\section{Impact of Interactions}

\section{Interaction between sugar beet varieties and planting densities}

The interaction between sugar beet varieties and planting densities significantly affected on root length, root and recoverable sugar yields/ fad., (Fig. 1A, B and C). It could be noticed that, root length of both Rass Poly and Panther was significantly and gradually decreased due to increasing planting density up to 42000 plants/ fad., while, root length of Pleno variety did not show significant response to varying planting densities. The longest root average was obtained by Pleno followed by Panther then Rass Poly with 28000 or 33600 plants/fad., while under the high planting density (42000 plants/fad.), Panther and Rass Poly had equal root length being lower than that of Pleno (Fig. 1A).

There is an evident from Fig. 1B that root yield of Rass Poly and Pleno varieties was significantly increased by increasing planting density up to 42,000 plants/fad., while root yield of Panther was responded only to increasing plant density up to 33,600 plants/fad. Under the middle planting density $(33,600$ plants/fad.) the highest root yield was obtained by Pleno followed by Panther then by Rass Polly, while under lower or higher planting densities, Panther and Rass Poly had equal root yield being lower than that of Pleno.

For Panther and Pleno sugar beet varieties, it was always true that, increasing planting density from 28000 to 33600 and 42000 plants/fad., gradually increased recoverable sugar yield/fad., (Fig. 1 C), while, recoverable sugar yield of Rass Poly was not significantly increased unless planting density was increased from 28 to 42 thousand plants/fad. Generally, Rass Poly and Panther varieties had equal recoverable sugar yield/fad., being lower than that obtained by Pleno variety. It could be included that, the maximum recoverable sugar yield ( 8.25 ton/ fad.) was achieved by Pleno variety with 42,000 plants/ fad.

\section{Interaction between sugar beet varieties and $K$ fertilizer levels}

Root length and diameter were significantly affected by the interaction between sugar beet varieties and $\mathrm{K}$ fertilizer levels. It is an evident from Fig. 2 A that root length of Rass Poly and Pleno varieties, significantly increased by raising $\mathrm{K}$ fertilizer level up to $24 \mathrm{~kg} \mathrm{~K}_{2} \mathrm{O} /$ fad., while root length of Panther variety showed significant response to $48 \mathrm{~kg} \mathrm{~K}_{2} \mathrm{O} / \mathrm{fad}$. Under without $\mathrm{K}$ application, Pleno variety surpassed the other two tested varieties in root length with no significant differences between Panther and Rass Poly in this respect. While under the application of either 24 or $48 \mathrm{~kg} \mathrm{~K}_{2} \mathrm{O} / \mathrm{fad}$., the longest roots was obtained by Pleno followed by Panther then by Rass Poly.

Root diameter of Rass Poly and Panther was increased by raising $\mathrm{K}$ fertilizer level up to 24 $\mathrm{kg} \mathrm{K}_{2} \mathrm{O}$ /fad., while, root diameter of Pleno did not significantly increased unless $48 \mathrm{~kg} \mathrm{~K}_{2} \mathrm{O}$ / fad., were applied. The longest root diameter was obtained from Pleno followed by Panther and Rass Poly with no significant differences between Pleno and Panther under the application of $24 \mathrm{~kg} \mathrm{~K}_{2} \mathrm{O} /$ fad., (Fig. $2 \mathrm{~B}$ ). 

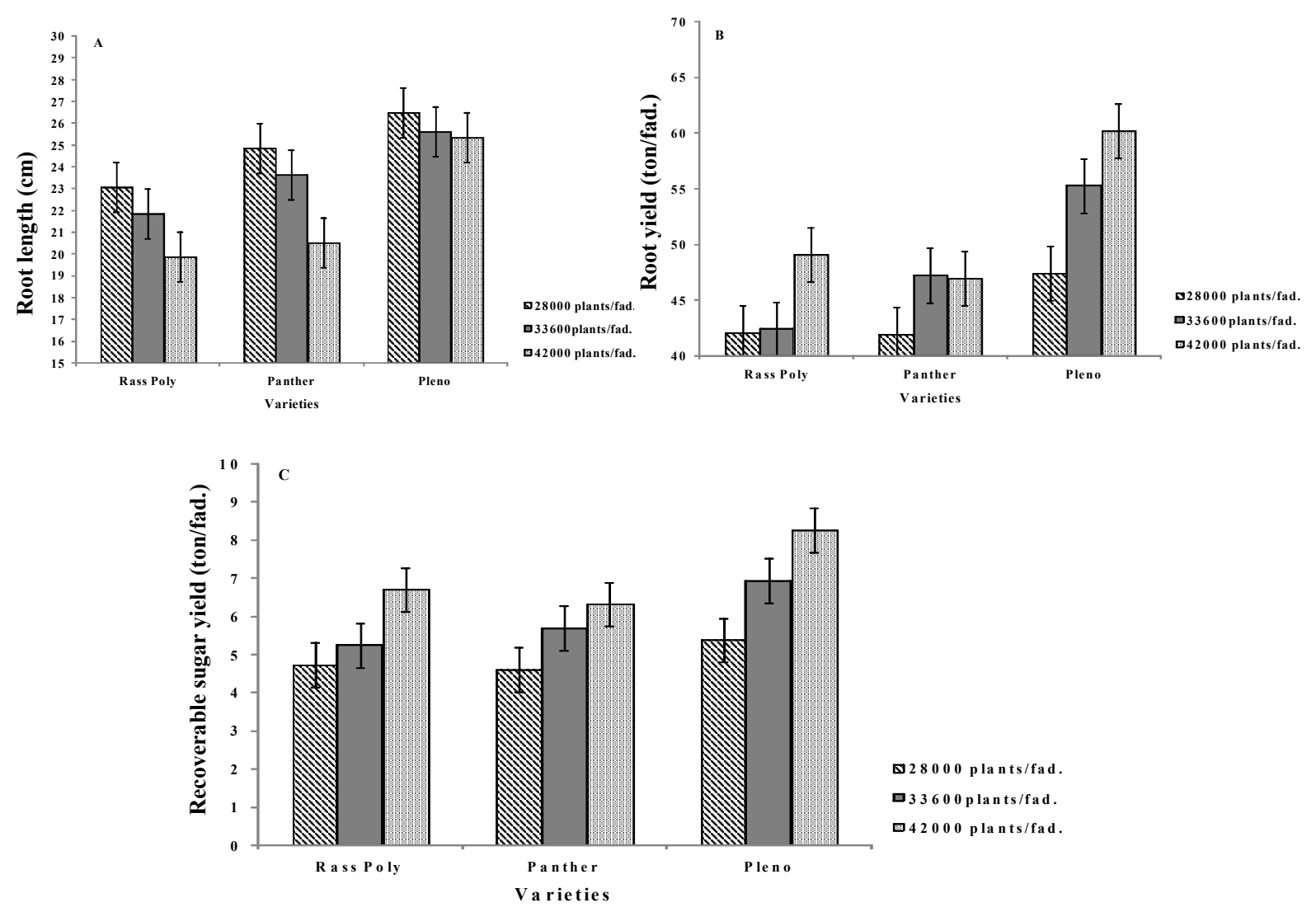

Fig. 1. Effect of the interaction between sugar beet varieties and planting densities on root length (A), root yield (B) and recoverable sugar yield (C)
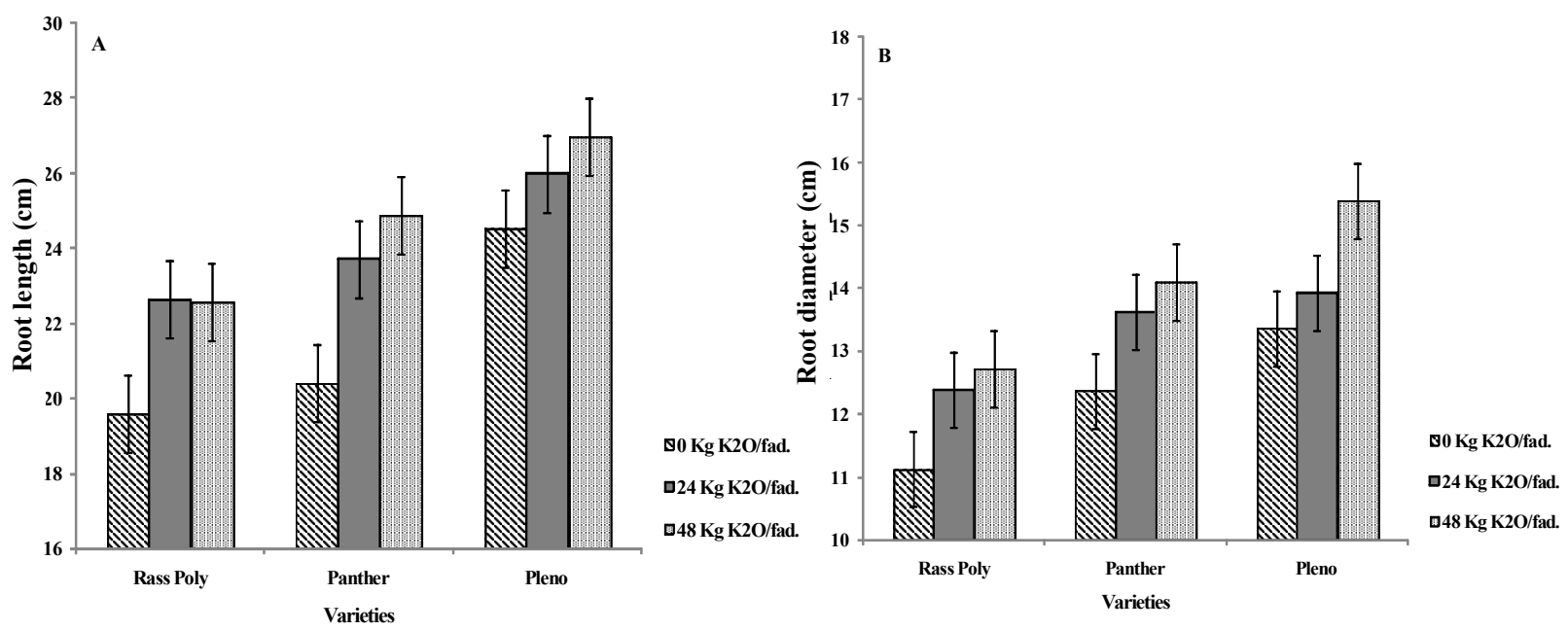

Fig. 2. Effect of the interaction between sugar beet varieties and $\mathrm{K}$ fertilizer levels on root length (A) and root diameter (B) 
Interaction between plant densities and $K$ fertilizer levels

Regardless planting densities effect, extractable sugar (\%) showed significant response to $\mathrm{K}$ application just to $24 \mathrm{~kg} \mathrm{~K}_{2} \mathrm{O} /$ fad. Under the low planting density (28000 plants/fad.) root and extractable sugar yields/fad., were significantly increased due to raising $\mathrm{K}$ fertilizer level up to $48 \mathrm{~kg} \mathrm{~K}_{2} \mathrm{O} /$ fad., while, under the higher planting densities it responded only to increasing $\mathrm{K}$ level up to $24 \mathrm{~kg} \mathrm{~K} \mathrm{~K}_{2} \mathrm{O} / \mathrm{fad}$. Regardless potassium fertilizer levels, extractable sugar (\%), root and recoverable sugar yields were significantly increased due to raising planting density up to 42000 plants/fad., (Fig 3 A, B and C).

\section{Conculusion}

It could be recommended from the results of obvious experimental field and technological analysis of sugar beet grown in sandy soil, that the maximum root and recoverable sugar yields/fad., could be obtained by planting Pleno variety with the dense planting of 42000 plants/ fad., and applying $24 \mathrm{~kg} \mathrm{~K}_{2} \mathrm{O} /$ fad.

It is worthy to mention that these results were obtained for growing sugar beet in newly reclaimed poor fertile sandy soil under drip irrigation, which encourage planting this crop under this conditions.

\section{Acknowledgment}

The author record his deepest gratitude and thanks to Zagazig University for providing facilities through funding the Project titled: Improvement productivity and quality of sugar beet under newly cultivated sandy soil.
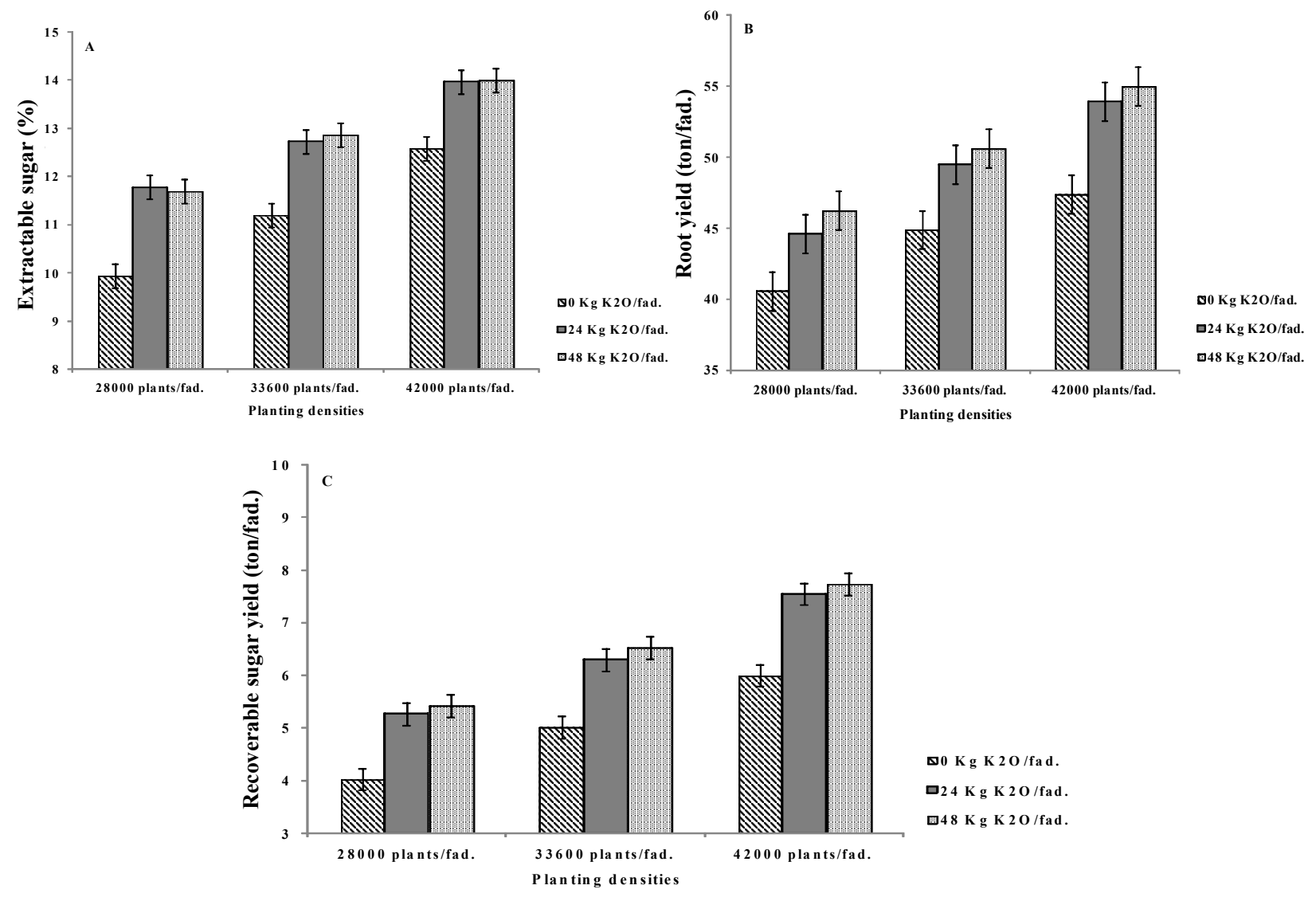

Fig. 3. Effect of the interaction between planting densities and $\mathrm{K}$ fertilizer levels on extractable sugar (\%) (A), root yield (B) and recoverable sugar yield (C) 


\section{REFERENCES}

Abdelaal, K.A.A., S.A. Badawy and S.M.M. Neana (2015). Effect of foliar application of microelements and potassium levels on growth, physiological and quality characters of sugar beet (Beta vulgaris L.) under newly reclaimed soils. J. Plant Prod., Mansoura Univ., 6 (1): 123 -133.

Abdel-Motagally, F.M. and K.K. Attia (2009). Response of sugar beet plants to nitrogen and potassium fertilization in sandy calcareous soil. J. Agric. and Bio., 11: 695-700.

Abo- Shady, Kh.A., S.M.M. Hilal, E. El-M. ElSheref and M.F.M. Ibrahim (2010). Yield and quality of sugar beet crop as affected by irrigation intervals, cultivars and potassium fertilization in North Delta. J. Agric. Res., KafrelSheikh Univ., 36 (4): 361-376.

Al-Sayed, H.M. and A.S. Attaya (2015). Effect of seed treatment and weed control methods on yield and quality of some sugar beet varieties. Zagazig J. Agric. Res., 42 (5): 939951.

Aly, E.F.A., A.H.S.A. Al-Labbody, M.E.R. Mekkei and E.A.M. El-Haggan (2012). Effect of hill spacing and cease irrigation before harvesting on some sugar beet varieties under sandy soils. J. Plant Prod., Mansoura Univ., 3 (6): 1039-1047.

AOAC (2005). Official Methods of Analysis of AOAC International. $16^{\text {th }}$ Ed. Washington, DC. USA.

Azzazy, N.B., N.M.S. Shalaby and A. M. Abd El-Razek (2007). Effect of planting density and days to harvest on yield and quality of some sugar beet varieties under Fayoum Governorate condition. Egypt. J. Appl. Sci., 22 (12A): 101-114.

Devillers, P. (1988). Prevision du sucre melasse. Sucrerie Francaise, 129: 190-200. (C.F. "The Sugar Beet Crop" D.A. Cook and R.A. Scott (Ed.). 571-617.Chapman and Hall, Lincoln.).

Dexter, S.T., M.G. Frankes and F.W. Snyder (1967). A rapid and practical method of determining extractable white sugar as may be applied to the evaluation of agronomic practices and grower deliveries in the sugar beet industry. J. Ame. Soc., Sugar beet Technol., 14: 433-454.

El-Bakary, H.M.Y. (2006). Studies on yield and quality characters of some sugar beet varieties. M.Sc. Thesis, Fac. Agric., AlAzhar Univ. Egypt.

El-Geddawy, I.H., A. M. A. EL Shafai and N. B. Azzazy (2006). Yield and quality of some sugar beet varieties as affected by planting densities and nitrogen fertilization. J. Agric. Sci. Mansoura Univ., 31(1): 43-54.

El-Ghareib, E.A., M.A. El-Hawary, A.M.A. ElShafai and Y.E.E. El- Rayess (2012). Effect of farmyard manure, plant density and biofertilizer treatments on growth and yield of sugar beet. J. Plant Prod., Mansoura Univ., 3 (7): 2173-2187.

El-Hity, M.A., M.S. Abdel-Atty, H.A. El-sharif and O.M. Hamed (2014). Sugar beet productivity as influenced by two commercial nutrient compounds under two levels of plant density. J. Plant Prod., Mansoura Univ., 5 (1): 53-67.

El-Sarag, E.I. (2009). Maximizing sugar beet yield, quality and water use efficiency using some agricultural practices under North Sinai conditions. Bulletin Fac., Agric. Cairo Univ., 60 (2): 155-167.

El-Sarag, E. I. and S. H. Moselhy (2013). Response of sugar beet quantity and quality to nitrogen and potasium fertilization under sandy soils conditions. Asian J. Crop Sci., 5 (3): 295-303.

El-Sheikh, S.R.E.; K.A.M. Khaled and S.A.A.M. Enan (2009). Evaluation of some sugar beet varieties under three harvesting dates. J. Agric. Sci. Mansoura Univ., 34 (3): 1559-1567.

Enan, S. A. A. M., E. F. A. Aly and A. I. Badr (2016). Effect of humic acid and potassium on yield and quality of some sugar beet varieties in sandy soil. J. Plant Prod., Mansoura Univ., 7 (2): 289 -297.

FAOSTAT, D. (2016). Food and agriculture organization of the United Nations. Statistical database.

Gomez, N.K. and A.A. Gomez (1984). Statistical Procedures for Agricultural 
Research. $2^{\text {nd }}$ Ed. John Wiley and Sons, New York, USA, 68.

Leilah, A., M. Badawi, E. Said, M. Ghonema, and M. Abdou (2005). Effect of planting dates, plant population and nitrogen fertilization on sugar beet productivity under the newly reclaimed sandy soils in Egypt. Sci. J. King Faisal Univ. (Basic and Appl. Sci.), 6 (1): 95-110.

Masri, M.I. (2008). Effect of nitrogen level and planting density on sugar beet yield and its attributes. Egypt . J. Agron., 30 (2): 119-136.

Mehrandish, M., M.J. Moeini and M. Armin (2012). Sugar beet (Beta vulgaris L.) response to potassium application under full and deficit irrigation. Euro. J. Exp. Bio., 2 (6): 2113-2119.

Merwad, A.M.A. (2016). Efficiency of potassium fertilization and salicylic acid on yield and nutrient accumulation of sugar beet grown on saline soil. Commun. Soil. Sci. Plant Anal., 47: 1184-1192.

Mohamed, H.F. (2002). Chemical and technological studies on sugar beet. Ph.D. Thesis, Fac. Agric., Minia Univ., Egypt.

MSTAT-C. (1991). A Microcomputer program for the design, management and analysis of agronomic research experiment. MSTAT Develop. Team, Michigan State Univ., East Lansing.

Mubarak, M.U., M. Zahir, M. Gul, M. Farooq and A. Wakeel (2016). Sugar beet response to potassium fertilizer under water sufficient and water deficient conditions. Electronic International Fertilizer Correspondent (e-ifc), 46: 30-32.

Nafei, A.I., A.M.H. Osman and M.M. El-Zeny (2010). Effect of plant densities and potassium fertilization rates on yield and quality of sugar beet crop in sandy reclaimed soils. (Beta vulgaris L.). J. Plant Prod., Mansoura Univ., 1 (2): 229-237.

Neana, S.M.M. and K.A.E. Abd El Hak (2014). Response of sugar beet for distance between hills and nitrogenous levels under different sowing dates. J. Adv. Agric. Res., Fac. Agric. Saba Basha., 19 (1): 126-136.
Osman, A.M.H., G.S. El-Sayed, M.S.H. Osman and K.S. El-Sogheir (2003). Soil application of some microelements with relation to yield and quality of sugar beet varieties. Ann. Agric. Sci., Moshtohor, 41 (3): 1071-1088.

Rengel, Z. and P. Damon (2008). Crops and genotypes differ in efficiency of potassium uptake and use. Physiol. Plant., 133(4):624-636.

Safina, S.A. and E.M. Abdel Fatah (2011). Response of three sugar beet varieties to compost, mineral nitrogen fertilizer and their combinations under sandy soil conditions IIYield and quality. Bull. Fac. Agric., Cairo Univ., 62 (4): 447-456.

Sarhan, H.M., M.A.E. Abdou and H.M. AlSayed (2012). Effect of planting systems, plant density and nitrogen fertilizer levels on productivity and quality of sugar beet. J. Plant Prod., Mansoura Univ., 3 (10): $2567-$ 2580.

Shalaby, N., A. Osman and A. Al-labbody (2011). Relative performance of sugar beet varieties under three plant densities in newly reclaimed soil. Egyptian J. Agric. Res., 89 (1): 291-299.

Steel, R.G.D., J.H. Torrie and D.H. Dickey (1997). Principles and Procedures of Statistics. A. Biometrical Approach, $3^{\text {rd }}$ Ed. Mc Graw Hill. Book Co. New Yourk, 172177.

Ulgen, A., A.U. Gokman, S.H. Herden and H. Cengiz (2009). Development of a $635 \mathrm{~nm}$ Laser Diode Spectrometer for Continous Monitoring of Amino Nitrogen in the Sugar Beet Industry. Instrumentation Sci. and Techno., 37(2): 218-229.

Varga, I., A. Kristek and M. Antunović (2015). Growth analysis of sugar beet in different sowing density during vegetation. Poljoprivreda, 21: 28-34.

Zengin, M., G. Fatma, M.Y. Atilla and S. Gez Gin (2009). Effect of potassium, magnesium and sulphur containing fertilizers on yield and quality of sugar beets (Beta vulgaris L.). Turk J. Agric., 33: 495-502. 


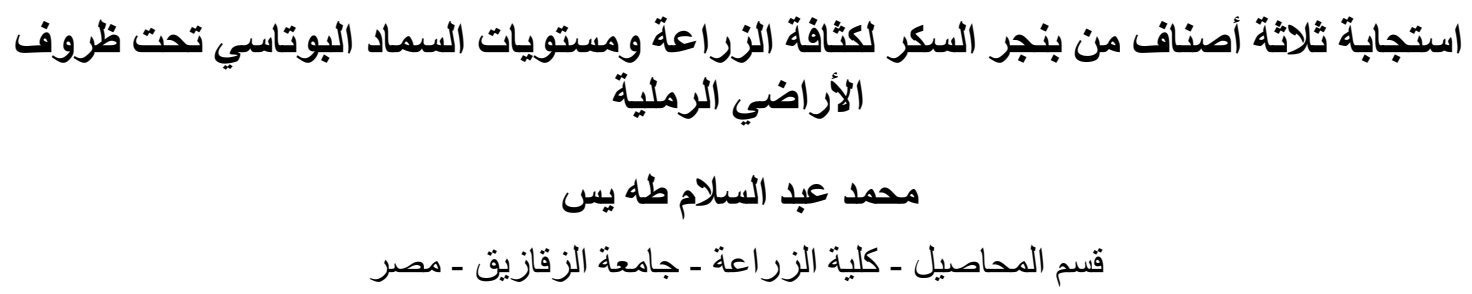

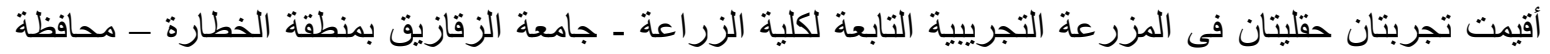

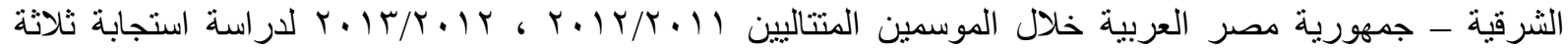

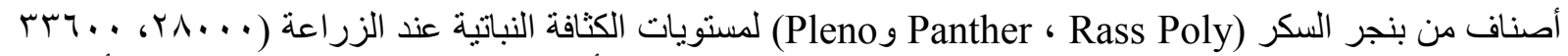

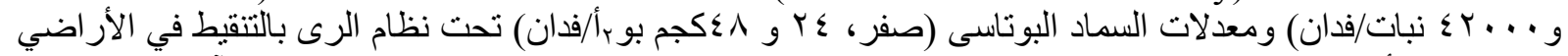

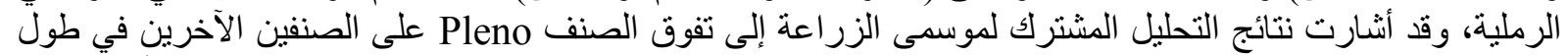

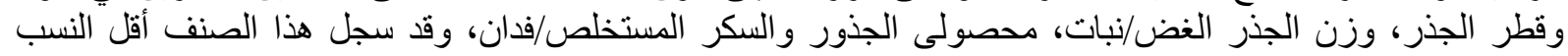

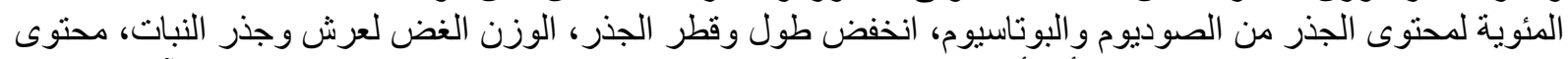

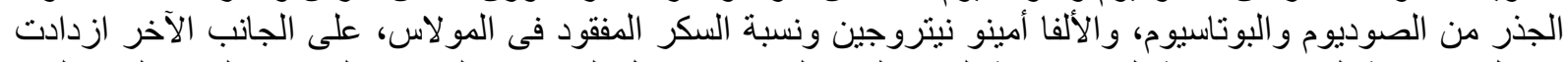

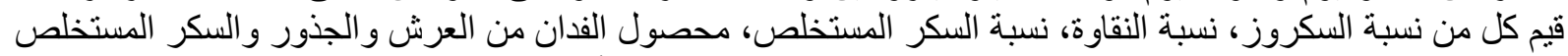

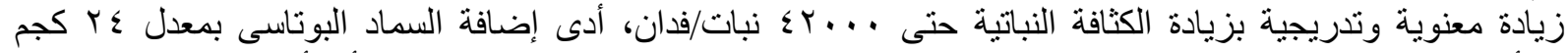

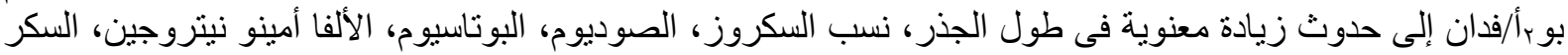

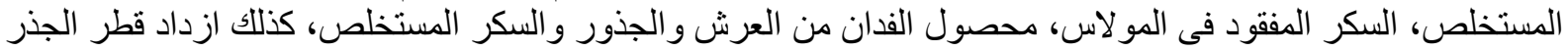

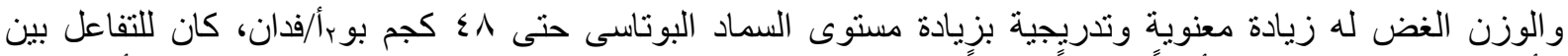

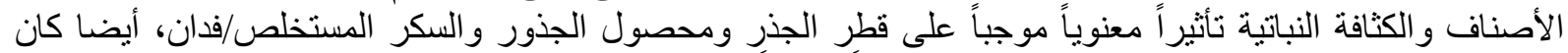

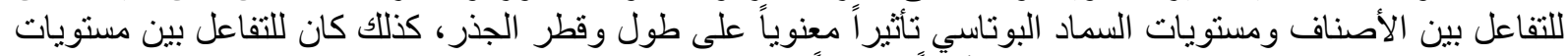

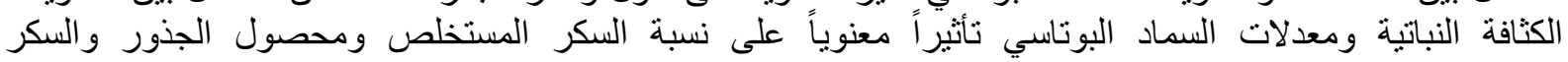

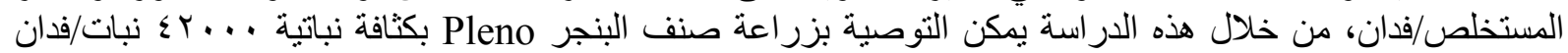

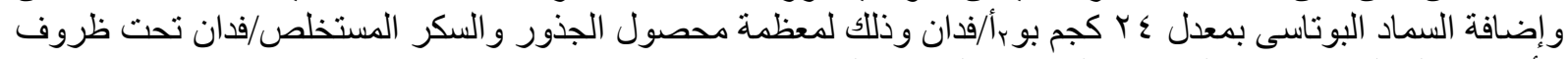

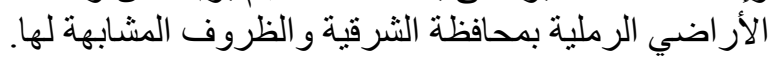

